\title{
A preceptoria na rede básica da Secretaria Municipal de Saúde do Rio de Janeiro: opinião dos profissionais de Saúde
}

\author{
Student supervision in primary care clinics in \\ the Rio de Janeiro City Health Department: \\ opinions by healthcare professionals
}

\author{
Anete Trajman ${ }^{\mathrm{I}}$ \\ Naima Assunção ${ }^{\mathrm{I}}$ \\ Monique Venturi ${ }^{\mathrm{I}}$ \\ Diogo Tobias ${ }^{\mathrm{I}}$ \\ Walria Toschi ${ }^{\mathrm{I}}$ \\ Victoria Brant ${ }^{\mathrm{II}}$
}

PALAVRAS-CHAVE

- Atenção primária à saúde.

- Educação médica.

- Tutoria.

- Motivação.
Recebido em: 06/06/2007

Reencaminhado em: 14/01/2008

Aprovado em: 18/03/2008
REVISTA BRASILEIRA DE EDUCAÇÃO MÉDICA

\begin{abstract}
R E S U M O
A formação de profissionais de saúde com as competências para prestação de cuidados básicos deve ser iniciada na graduação. Entretanto, o treinamento na rede básica de saúde (RBS) ainda encontra barreiras nas esferas docente, discente e nas unidades das Secretarias Municipais de Saúde (SMS). Considerando a relevância do papel dos preceptores na formação dos estudantes da área da saúde, avaliamos a opinião dos profissionais de saúde da RBS da SMS do Rio de Janeiro sobre a atividade de preceptoria. Um questionário foi respondido por 351 profissionais de saúde de 13 das 67 unidades da RBS. Destes, 77\% consideram que a preceptoria faz parte das atribuições do profissional e 61\% gostariam de assumir esta tarefa. Várias dificuldades foram apontadas, incluindo problemas estruturais e de recursos humanos. Os resultados responsabilizam, de alguma forma, as instituições de ensino superior (IES) e o Estado pela pouca valorização e estímulo às ações de preceptoria, na medida em que apontam a necessidade de rever as condições de trabalho e de ensino na RBS. Destacam-se a melhoria dos salários e da infraestrutura e a oportunidade de capacitação profissional, o que implica parcerias efetivas entre as IES e as SMS.
\end{abstract}

\section{A B S T R A C T}

Training of health professionals with the necessary skills in primary care should begin during undergraduate medical education. However, training in the primary care network still faces obstacles among faculty, students, and the primary care clinics operated by municipal health departments. Considering the relevant role of supervisors in training students in health-related fields, we investigated the opinions of healthcare professionals in the primary care network in Rio de Janeiro concerning their student supervision activities. A total of 351 health professionals from 13 of the 67 primary care clinics answered a questionnaire. Of these, $77 \%$ felt that student supervision is part of their responsibilities, and $61 \%$ said they would like to play this role. Various difficulties were identified, including structural and human resources problems. According to the findings, medical schools and the government are responsible for the under-valuing of student supervision, emphasizing the need to review the working and teaching conditions in the primary care network. The results also highlight improvements in wages and infrastructure and the opportunity for professional advancement, requiring effective partnerships between medical schools and the Municipal Health Department.

\footnotetext{
I Universidade Gama Filho, Rio de Janeiro, Brasil.

${ }^{I I}$ Universidade Federal do Rio de Janeiro. Núcleo de Tecnologia de Ensino em Saúde, Rio de Janeiro, Brasil.
} 


\section{INTRODUÇÃO}

Nas últimas décadas, a Atenção Básica à Saúde (ABS) tornou-se um tema especialmente relevante e resultou na reformulação de diferentes sistemas nacionais de saúde. A universalidade e a garantia de acesso por meio dos cuidados primários de saúde têm sido preconizadas em vários países do mundo como forma de alcançar maior equidade e satisfação das expectativas dos usuários ${ }^{1}$.

A definição e os princípios que embasam atualmente a ABS superaram a visão de que esta seria meramente um dos níveis do sistema de saúde ou um campo específico de atuação. Ao contrário, a ABS é considerada como reorganizadora das práticas individuais e coletivas, e reorientadora do Sistema Único de Saúde, compreendendo a garantia da integralidade em suas duas dimensões básicas de abrangência e coordenação². Estas reformulações mais recentes de base filosófica, metodológica e organizacional trouxeram para as instituições de ensino médico e das demais áreas da saúde novos desafios para o ensino de graduação. A formação de profissionais de saúde com as competências para prestação de cuidados básicos deve se iniciar na graduação, considerando a ABS não apenas um campo de práticas, mas também um corpo de conhecimentos que exige abordagens disciplinar e pedagógica inteiramente novas. Ao assumir as múltiplas tarefas relacionadas aos seus princípios, caberá ao egresso a assistência de forma personalizada e continuada a indivíduos e famílias em seu contexto comunitário e territorial. É nestes cenários que terão a oportunidade de participar das atividades de promoção de saúde e resolver $80 \%$ dos problemas de saúde da população, justificando-se, pois, a ênfase na ABS, para o que é necessária a convergência de todos os esforços, como apontado por Campos ${ }^{3}$ :

Há uma recomendação curricular genérica de que a formação médica busque uma variação de cenários para o ensino prático. Na mesma linha recomenda-se a inserção precoce do estudante em atividades práticas. Além disso, se na rede básica se espera sejam resolvidos $80 \%$ dos problemas de saúde da população, se aceitamos que as intervenções no território são de grande complexidade, e se ainda acrescentamos a isso que grande parte da população brasileira vive na pobreza, teremos claras evidências técnicas e éticas de que nossas escolas médicas devem formar um profissional competente para intervir nessa realidade. Nesse sentido, a rede básica é um campo de práticas potencial e necessário, no qual os vários cursos de formação de profissionais de saúde deverão inserir seus estudantes.
Em resposta a este desafio, o Ministério da Educação (MEC) elaborou e publicou as Diretrizes Curriculares Nacionais para os cursos da área da saúde em 2001 e 2002 ${ }^{4-6}$, em que recomenda a realização do treinamento em diferentes cenários e níveis hierárquicos de atenção, incluindo a ABS. O treinamento do estudante da área da saúde, tendo em vista a necessidade de reformular a orientação profissional, tornou-se um importante desafio, posto que grande parte das IES ainda segue o modelo hospitalocêntrico. A fim de incentivar a transformação das escolas médicas, de enfermagem e de odontologia, os Ministérios da Saúde e da Educação publicaram, então, Portarias Interministeriais que marcaram o início de uma nova era, destacando-se, entre elas, a criação dos Polos de Educação Permanente em Saúde (PEPs) ${ }^{7}$ e o lançamento dos Programas de Incentivo às Mudanças Curriculares no Ensino Médico (Promed), em 2002, e o de reorientação profissional, o Pró-Saúde ${ }^{8}$, em 2005.

Entretanto, para implementar novas políticas de educação em saúde, é necessária a integração estreita entre os serviços de saúde e a academia, considerando, inclusive, que o Artigo no 200, inciso III, da Constituição Federal de 1988 estabelece que a formação de recursos humanos para a saúde é atribuição do Sistema Único de Saúde (SUS) ${ }^{9}$. Embora não haja estudos sistematizados sobre o tema, e apesar de constituir um dispositivo constitucional e uma necessidade que vem sendo reconhecida por estudantes e profissionais, tanto dos serviços quanto da academia, há percepção de gestores e educadores na área da saúde de séria resistência de docentes, discentes e profissionais de saúde para assumir este treinamento na rede básica de atendimento.

Acrescenta-se aqui a questão do recente envolvimento dos profissionais do SUS com atividades de supervisão/orientação de estudantes de graduação da área da saúde. Esse envolvimento - que vem sendo nomeado preceptoria - não só é recente como exige o acréscimo de uma formação/aculturação pedagógica para além das funções técnicas que lhe são atribuídas.

Paralelamente, a inserção dos estudantes de graduação na rede traz para debate algumas questões de ordem pedagógica, uma vez que pressupõe um trabalho de mediação entre teoria e prática a ser realizado por docentes das instituições formadoras e também por tutores ou colaboradores - trabalhadores das unidades de saúde que possam atuar como preceptores destes estudantes. Isto nos remete aos sérios problemas vividos por esses trabalhadores no que se refere às políticas de recursos humanos, às condições de precarização vividas no ambiente de trabalho e à inexistência de oportunidades para a educação continuada, entre outros.

A maioria dos profissionais da rede não encontra apoio institucional ou oferta de oportunidades para acesso a cursos de for- 
mação especializada em saúde da família, em saúde coletiva, ou mesmo para o exercício de uma clínica ampliada de cunho generalista, conforme as prioridades estabelecidas para o setor. Este quadro se agrava ainda mais na ocasião da entrada dos estudantes em estágios curriculares nas unidades de saúde, o que torna recomendável que as instituições formadoras proponham projetos de integração docente-assistencial com as Secretarias Municipais, de modo a contribuir para minimizar o afastamento de suas respectivas ações. A viabilização destes estágios é, sem dúvida, uma estratégia para formar profissionais de saúde com perfil mais adequado às diretrizes político-sanitárias, com benefício direto à população assistida pelo SUS.

Com base nestas questões e considerando a relevância do papel dos preceptores na formação dos estudantes do curso de Medicina, o objetivo do estudo foi avaliar a opinião dos profissionais de saúde da rede básica municipal do Rio de Janeiro sobre a atividade de preceptoria, como parte de um projeto mais amplo de avaliação da integração docente-assistencial.

\section{MÉTODO}

\section{Tipo e local de estudo}

Entre janeiro de 2005 e maio de 2006, desenvolveu-se um estudo transversal, do tipo inquérito, baseado em questionário autoaplicável, em 13 unidades de atendimento básico situadas em diferentes áreas programáticas, selecionadas por conveniência dos pesquisadores entre as 67 unidades existentes no município do Rio de Janeiro. As unidades foram selecionadas de acordo com contato prévio feito pelos pesquisadores (AT e WT) com diretores interessados na realização da pesquisa em suas unidades, após breve explicação dos objetivos e métodos. Todas as unidades já recebiam, à época do estudo, estagiários da área de saúde.

\section{Instrumento}

O questionário - oferecido a todos os profissionais de saúde presentes no dia das visitas dos estudantes às unidades e respondido por aqueles que concordaram em participar - continha perguntas objetivas sobre dados sociodemográficos, profissão, tipo de vínculo na SMS e outros vínculos empregatícios, incluindo os de instituição de ensino superior (IES). Também foi perguntado se o profissional considerava a atividade de preceptoria parte de suas atribuições e se ele gostaria de orientar estudantes. Havia, ainda, perguntas abertas sobre problemas para o treinamento de estudantes e para o exercício da profissão em suas unidades e sobre pontos positivos e negativos da formação de estudantes na unidade, reconhecidos para os profissionais, estudantes e para o próprio serviço. Finalmente, formulamos perguntas fechadas sobre os incentivos para participar da supervisão como preceptores. Entre 17 opções oferecidas, possíveis incentivos para desenvolver a atividade de preceptoria foram classificados pelo profissional como irrelevante, desejável, importante ou fundamental.

\section{Análise dos dados}

Para o escore de incentivos, um valor de 0 a 3 foi atribuído às respostas (0 para irrelevante, 1 para desejável, 2 para importante, 3 para fundamental), e a soma dos valores calculada em um escore de importância. Para comparação entre médicos e profissionais de outras categorias, as respostas foram agrupadas em importante/fundamental versus desejável/irrelevante.

As respostas abertas (pontos positivos e negativos) foram analisadas agrupando-se as opiniões em categorias relativas às condições de trabalho (incluindo as condições para o usuário), ao aprendizado, e às colaborações interinstitucionais.

Os dados foram armazenados e analisados no programa SPSS versão 10.0. A razão das chances (OR) foi utilizada como medida de associação entre as variáveis na análise univariada. Para as variáveis associadas com $p$ valor $<0,20$, a OR ajustada (aOR) e o seu intervalo de confiança de $95 \%$ foram calculados utilizando-se análise multivariada, com modelo de regressão logística. Na análise multivariada, um $p$ valor $<0,05$ foi considerado estatisticamente significativo.

O estudo foi aprovado pelo Comitê de Ética em Pesquisa do Hospital Municipal Souza Aguiar da Secretaria Municipal de Saúde do Rio de Janeiro (SMS-RJ), antes da constituição do comitê próprio da SMS. Somente foram incluídas unidades cujas direções expressaram seu acordo por meio da assinatura de autorização.

\section{RESULTADOS}

Entre 426 profissionais presentes nos dias do inquérito, 351 $(82,4 \%)$ responderam ao questionário. A maior parte dos que recusaram responder alegava falta de tempo. A Tabela 1 contém as características sociodemográficas e profissionais dos respondentes. Entre os 347 que responderam à pergunta fechada "faz parte do papel do profissional do SUS orientar estudantes?", 267 (76,9\%) responderam afirmativamente (Tabela 2). Dentre 322 que responderam à pergunta "você gostaria de supervisionar atividades práticas de estudantes?", 204 (61,4\%) responderam afirmativamente. Os problemas identificados nas respostas abertas com maior frequência foram: espaço físico reduzido (24,5\%), carência de recursos $(20,7 \%)$, falta de tempo dos profissionais $(14,4 \%)$ e despreparo profissional $(11,2 \%)$. Os pontos negativos e positivos identificados para estudantes, serviço e profissionais de saúde estão apresentados nas Tabelas 3 e 4 . O incentivo mais citado espontaneamente foi a remuneração por esta atividade $(44,5 \%)$. A soma dos escores dos incentivos encontra-se na Tabela 5 . 
Tabela 1

Características dos 351 profissionais de saúde da rede básica da SMS que responderam ao questionário autoaplicável - Rio de Janeiro, 2005-2006

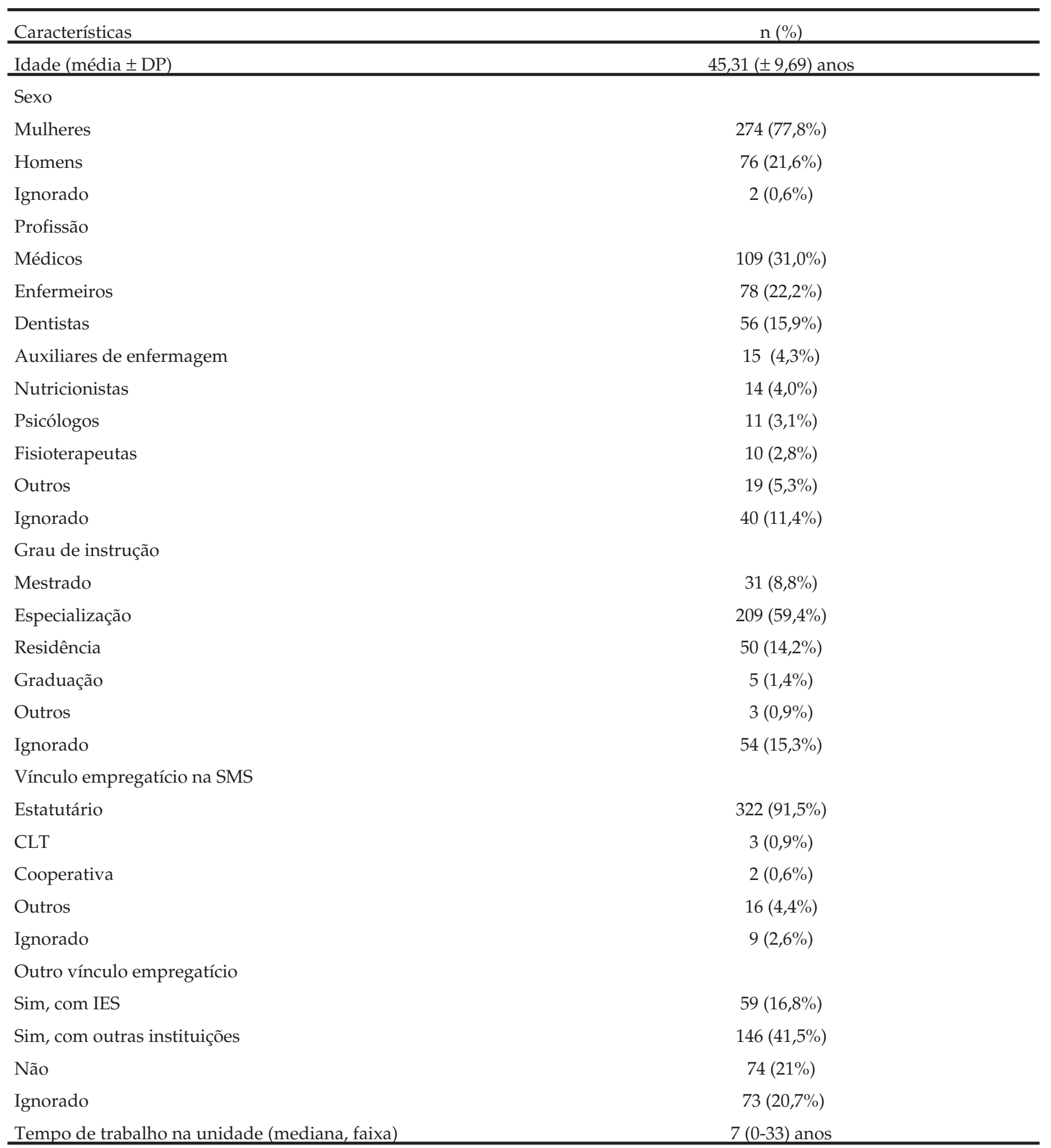


Tabela 2

Profissão dos 238 profissionais de saúde da SMS que responderam sim à pergunta "faz parte do papel do profissional do SUS orientar estudantes?" - Rio de Janeiro, 2005-2006.

\begin{tabular}{lc}
\hline Profissão & Resposta “Sim" n (\%) \\
\hline Nutricionista & $14 / 14(100 \%)$ \\
Farmacêutico & $5 / 5(100 \%)$ \\
Fonoaudiólogo & $5 / 5(100 \%)$ \\
Enfermeiro & $74 / 78(94,9 \%)$ \\
Auxiliar de enfermagem & $11 / 12(91,7 \%)$ \\
Dentista & $46 / 56(82,1 \%)$ \\
Fisioterapeuta & $7 / 10(70 \%)$ \\
Médico & $62 / 109(56,9 \%)$ \\
Psicólogo & $5 / 11(45,5 \%)$ \\
Outros & $9 / 9(100 \%)$ \\
\hline
\end{tabular}

Tabela 3

Pontos positivos para a atividade de preceptoria na unidade identificados pelos profissionais de saúde da rede básica da SMS do Rio de Janeiro, 2005-2006

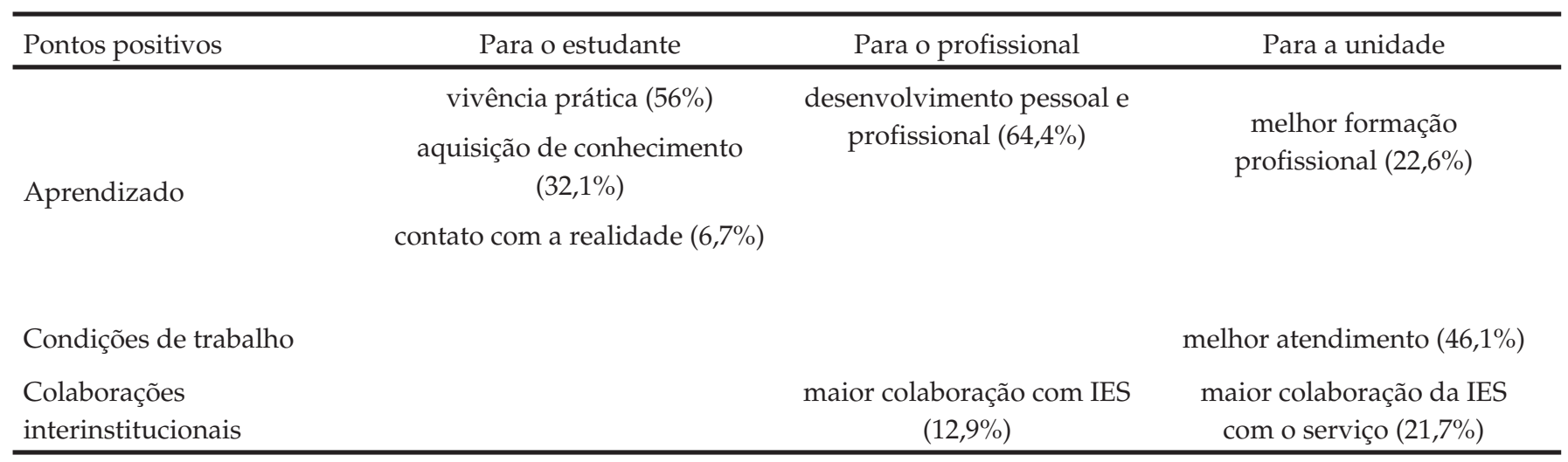

Quando comparados com as demais categorias profissionais, os médicos apontaram com frequência significativamente superior o agendamento de menor número de pacientes [OR $=$ $\left.5,42(3,11 ; 9,50), \mathrm{p}<10^{-4}\right]$ e passagens para participar de congres$\operatorname{sos}[\mathrm{OR}=2,03(1,18 ; 3,49), \mathrm{p}=0,009]$. Inversamente, os médicos apontaram com frequência significativamente inferior os seguintes incentivos: acesso aos periódicos assinados pela IES [OR $=0,49(0,29 ; 0,85), \mathrm{p}=0,006]$; aquisição de equipamentos para a unidade [OR $=0,48(0,27 ; 0,84), \mathrm{p}=0,006]$; melhoria da infraes- trutura da unidade [OR $=0,48(0,25 ; 0,90), \mathrm{p}=0,013]$; e melhoria da biblioteca da unidade $\left[\mathrm{OR}=0,42(0,25 ; 0,71), \mathrm{p}<10^{-4}\right]$.

As únicas variáveis significativamente associadas à resposta afirmativa para "faz parte do papel do profissional do SUS orientar estudantes?" foram a titulação [aOR = 1,65 $(1,02 ; 2,67), \mathrm{p}=$ $0,042]$ e pertencer a outra categoria que não médicos $[\mathrm{aOR}=4,96$ $\left.(2,76-8,90), \mathrm{p}<10^{-4}\right]$. Os profissionais com outro vínculo em IES responderam afirmativamente à pergunta "você gostaria de supervisionar atividades práticas de estudantes?" com maior frequência $\left[\mathrm{OR}=10,15(3,03 ; 33,93), \mathrm{p}<10^{-4}\right]$. 
Tabela 4

Pontos negativos para a atividade de preceptoria na unidade identificados pelos profissionais de saúde da rede básica da SMS do Rio de Janeiro, 2005-2006.

\begin{tabular}{|c|c|c|c|}
\hline Pontos negativos & Para o estudante & Para o profissional & Para a unidade \\
\hline \multirow{6}{*}{ Aprendizado } & sem pontos negativos $(33,3 \%)$ & \multirow[t]{6}{*}{ sem pontos negativos $(26,7 \%)$} & \multirow[t]{6}{*}{ sem pontos negativos $(58,8 \%)$} \\
\hline & $\begin{array}{c}\text { conviver com as deficiências } \\
\text { do serviço }(49,1 \%)\end{array}$ & & \\
\hline & falta de profissionais & & \\
\hline & preparados $(12,3 \%)$ & & \\
\hline & contato com a realidade & & \\
\hline & $(5,3 \%)$ & & \\
\hline \multirow{4}{*}{ Condições de trabalho } & & \multirow{4}{*}{$\begin{array}{l}\text { sobrecarga de trabalho }(43,3 \%) \\
\text { interferência na relação } \\
\text { médico-paciente }(15,6 \%) \\
\text { divisão do espaço físico }(11,1 \%)\end{array}$} & $\begin{array}{l}\text { constrangimento do paciente } \\
\qquad(176 \%)\end{array}$ \\
\hline & & & \\
\hline & & & consumo de recursos $(11,8 \%)$ \\
\hline & & & $\begin{array}{l}\text { alteração na rotina diária } \\
\qquad(5,9 \%)\end{array}$ \\
\hline $\begin{array}{l}\text { Colaborações } \\
\text { interinstituicionais }\end{array}$ & \multicolumn{3}{|c|}{ nenhum ponto negativo relativo às colaborações insterinstitucionais identificado } \\
\hline
\end{tabular}

Tabela 5

Soma do escore dos incentivos para a preceptoria classificados por 351 profissionais de saúde da rede básica da SMS do Rio de Janeiro, 2005-2006.

\begin{tabular}{lc}
\hline Incentivos & Soma dos escores \\
\hline Melhoria da infra-estrutura da unidade & 922 \\
Melhores salários & 920 \\
Liberação para participação em congresso & 906 \\
Liberação para cursar pós-graduação & 898 \\
Aquisição de equipamentos para unidade & 897 \\
Complemento salarial para tarefa de preceptoria & 888 \\
Incentivo à pesquisa na unidade & 883 \\
Capacitação didático-pedagógica & 875 \\
Acesso aos periódicos assinados pela IES & 875 \\
Capacitação na área de atuação & 873 \\
Acesso à biblioteca da IES & 846 \\
Acesso aos computadores da IES & 836 \\
Passagens para participação em congressos & 818 \\
Melhoria da biblioteca da unidade & 772 \\
Presença de preceptores da IES na unidade & 769 \\
Apoio psicopedagógico & 669 \\
Agendamento de menor número de pacientes & 667 \\
\hline
\end{tabular}




\section{DISCUSSÃO}

Este inquérito mostra que a maior parte dos profissionais tem interesse em desempenhar a função de preceptor. Entretanto, identificamos claramente o desinteresse de muitos nesta tarefa, principalmente entre médicos e psicólogos, mesmo entre aqueles que concordam com a importância desta atividade na formação do estudante. Os profissionais reconhecem, para o desenvolvimento das atividades de preceptoria, dificuldades na infraestrutura (espaço e recursos inadequados) e, em menor escala, problemas de recursos humanos (despreparo e falta de tempo).

Muitos consideram que o treinamento do estudante em condições precárias pode ser ruim, embora outros julguem que este é um ponto positivo, pois o prepara para as condições que encontrará na vida profissional. Em relação ao serviço, alguns temem pela qualidade da relação médico-paciente, o que, em parte, encontra fundamento. Mostram ainda preocupação com a formação dos profissionais de saúde, ao identificarem, entre os pontos negativos, o "despreparo" dos profissionais e, entre as dificuldades, a "carência" de recursos humanos.

Os profissionais com vínculo em IES responderam que gostariam de desempenhar a preceptoria com maior frequência. Possivelmente sentem maior segurança por já desenvolverem atividades de ensino. A maioria dos profissionais da rede não encontra apoio institucional ou oferecimento de oportunidades para acesso a cursos de formação especializada em saúde da família, em saúde coletiva ou mesmo para o exercício de uma clínica ampliada de cunho generalista, conforme as prioridades estabelecidas para o setor.

Esse quadro se agrava ainda mais na ocasião da entrada dos alunos em estágios curriculares nas unidades de saúde, o que torna no mínimo recomendável que as instituições formadoras proponham projetos de integração docente-assistencial com as Secretarias Municipais, de modo a contribuir para minimizar o afastamento de suas respectivas ações. A viabilização desses estágios é, sem dúvida, uma estratégia para formar profissionais de saúde com perfil mais adequado às diretrizes político-sanitárias, com benefício direto à população assistida pelo SUS. A formação dos preceptores deve ser uma função prioritária das IES, no que se refere tanto à atualização profissional quanto às funções de ensino. É esta a vocação das universidades e deve ser esta a sua principal contrapartida nos convênios com as SMS.

De forma coerente com os pontos negativos referidos, os incentivos à preceptoria mais frequentemente apontados dizem respeito à melhoria das condições de trabalho: melhor remuneração e capacitação dos profissionais, e melhoria da infraestrutura da unidade. Ressalta-se que, quando escolhem entre os incen- tivos, a melhor remuneração por suas atividades é mais importante do que o complemento salarial para a tarefa de preceptoria. Este é um ponto que nos parece particularmente interessante e coerente com a opinião maciça de que as atividades de preceptoria fazem parte de suas atribuições, podem e devem ser incorporadas no ambiente de trabalho como parte da estratégia de Educação Permanente. Ademais, a remuneração do profissional de saúde pela IES conveniada pode resultar em salários diferenciados na unidade, com consequente frustração dos profissionais não gratificados. $\mathrm{O}$ apoio psicopedagógico e a presença de preceptores da IES na unidade não foram tão importantes quanto a melhoria das condições de trabalho.

Curiosamente, o agendamento de menor número de pacientes foi o último na ordem de preferências, embora um número expressivo de profissionais tenha espontaneamente referido falta de tempo como um dos problemas para esta tarefa. De fato, embora a maior parte dos profissionais abordados tenha concordado em participar, a principal razão para a recusa foi a falta de tempo, o que pode ter resultado em um viés de seleção. Desta forma, é possível que a inclusão destes profissionais tivesse aumentado a porcentagem dos que veem a falta de tempo como dificuldade para a preceptoria e dos que gostariam de ter menor número de pacientes agendados. Embora último no ranqueamento, para os médicos este incentivo foi importante. A presença de estudantes nos ambulatórios gera a necessidade de maior tempo de consulta, pois o estudante precisa de mais tempo para completar a anamnese e o exame físico, e a consulta deve ser seguida de um tempo para discussão das hipóteses diagnósticas e da conduta. Portanto, apesar do baixo ranqueamento entre os incentivos, esta é uma questão que deve ser tratada com atenção nos acordos interinstitucionais, principalmente no que se refere aos estágios para estudantes de Medicina. A falta de tempo e a sobrecarga de trabalho apareceram, de fato, como dificuldades nas respostas abertas.

Os médicos, que mostraram menor interesse no acesso aos periódicos e na melhoria da biblioteca da unidade, possivelmente têm outras formas de acesso à atualização, por meio de assinatura de periódicos e acesso pela internet aos inúmeros sites de educação continuada. Entretanto, expressaram seu interesse na participação em congressos como forma de educação continuada.

As condições de infraestrutura também precisam ser adequadas para o acolhimento do estudante. O reduzido espaço e as precárias condições físicas foram apontados tanto na pergunta sobre as dificuldades quanto nos pontos negativos.

Apesar das dificuldades e dos pontos negativos apontados, muitos profissionais reconhecem vantagens na preceptoria. Os 
pontos positivos mais mencionados foram a possibilidade de seu próprio desenvolvimento profissional e a melhoria do atendimento. Estes pontos foram percebidos independentemente do tempo de trabalho, da titulação e do vínculo com algumas IES. Parece, portanto, que a preceptoria nas unidades básicas pode atender a alguns anseios dos profissionais, desde que o acordo entre as instituições leve em conta estas expectativas.

A escuta da fala destes profissionais em estudos que utilizam metodologias qualitativas, em que possam expressar livremente seus receios e anseios em relação à preceptoria, poderá enriquecer os achados que este estudo aponta. Neste sentido, estamos propondo um estudo baseado em grupos focais, constituídos por diferentes categorias profissionais, uma vez que se encontrou significativa diferença nas respostas de diferentes profissionais de saúde. No estudo proposto, poderemos aprofundar as questões do treinamento profissional, da infraestrutura dos serviços e do que percebem como sendo um possível fator dificultador da relação médico-paciente.

Do ponto de vista do interesse das IES, o treinamento do estudante nas condições do mundo real - as mesmas que encontrará no mercado de trabalho -, visto como uma desvantagem por alguns profissionais, pode constituir, na realidade, uma vantagem, uma vez que o SUS é, no Brasil, o maior empregador de profissionais de saúde, e as Diretrizes Curriculares recomendam o treinamento na atenção básica. Entretanto, o profissional precisa ser capacitado não apenas do ponto de vista da atualização profissional, como são habitualmente feitos os treinamentos da SMS, mas formados também na concepção de educação permanente. Os profissionais precisam estar aptos à autoeducação continuada, uma vez que o avanço do conhecimento científico resulta em mudanças constantes e rápidas das condutas preconizadas, exigindo que o profissional reveja criticamente as novas recomendações e diretrizes que surgem. Por outro lado, se serão responsabilizados pela avaliação do estudante, precisam ser capacitados para esta tarefa. Uma das mais difíceis atribuições da docência é a avaliação por competências, e não podemos esperar que o profissional de saúde desempenhe esta tarefa sem qualquer treinamento, como vem sendo realizado na prática. O conhecimento das Diretrizes Curriculares, dos objetivos do curso e do perfil do egresso de cada IES que estabelece parcerias com a unidade de saúde também precisa ser discutido com o profissional, para que este possa compreender qual o seu papel e sua responsabilidade na formação do estudante.

As parcerias entre IES e SMS precisam ser revistas para atender aos anseios dos docentes, discentes e profissionais, embora parceria implique redistribuição de poder, um processo sempre conflituoso, segundo Almeida. ${ }^{11}$ Nem sempre o que as IES entendem como necessidades de saúde da população significa, de fato, o que ela deseja, o que anseia; e quanto mais os atores se envolverem com as decisões, discutindo-as coletivamente, mais efetivo se tornará o benefício para todos.

O principal papel das IES deve ser o de reconhecer e capacitar o profissional interessado na atividade de preceptoria para que ele desempenhe com segurança e competência o que, na sua própria visão, faz parte de suas atribuições. E isso precisa ser discutido no âmbito dos serviços de saúde e nas IES:

[...] os profissionais dos serviços valorizam, de forma geral, os estímulos com os alunos e os professores. Estes, quando realizam práticas nos novos cenários, também valorizam as experiências adquiridas como meios de adequação dos conteúdos curriculares, propiciados pela incorporação de temas/problemas com maior relevância ${ }^{11}$.

Por outro lado, a melhoria das condições de trabalho deve ser considerada pelas autoridades competentes para que a rede básica possa, de fato, constituir um espaço adequado de treinamento dos estudantes. Um esforço conjunto das SMS, dos corpos docente e discente das IES e dos profissionais de saúde é o mínimo necessário para que tenhamos quadros eficientes e resolutivos na área da saúde no Brasil e, em especial, no fortalecimento do SUS.

Consideramos que a formação pedagógica de preceptores deva ter como meta compreender o que significa um processo dialético de ensino-aprendizagem, por meio da adoção de um modelo educativo e de perspectivas pedagógicas que superem a mera transmissão de conhecimentos e que levem os profissionais a extraírem das situações complexas e contraditórias de seus exercícios profissionais diários a possibilidade de superar obstáculos e construir alternativas de solução. Para assegurar aos preceptores um processo que contribua para uma reflexão sistemática e bem fundamentada sobre o modelo de atenção à saúde, é preciso uma estratégia educativa que favoreça uma perspectiva emancipadora. A reflexão crítica sobre os processos de trabalhomesmo em condições de alienação e de subordinação a uma lógica que dificulta o exercício profissional como prática criadora e capaz de dotar os sujeitos de satisfação - é uma condição necessária para ampliar as dimensões realizadoras do trabalho na saúde. Para tanto, é crucial que os profissionais elevem sua formação profissional e ético-política, objetivando transformar substantivamente essas condições adversas, e que possam realizar, 
no espaço do SUS, o conceito de saúde gestado na Reforma Sanitária que impulsionou originalmente o projeto universalista da reforma da saúde pública brasileira.

Por fim, os estudantes também precisam ser ouvidos, pois têm sua própria visão, receios e expectativas, que precisam ser conhecidos e pactuados à luz das necessidades e expectativas dos profissionais de saúde.

\section{AGRADECIMENTO}

Os autores agradecem à Prof. Eliana Claudia Ribeiro pela contribuição na concepção do questionário.

\section{REFERÊNCIAS}

1. Health Council of the Netherlands. European primary care. Draft report presented to the conference Shaping the EU Health Community, The Hague, 7-9 Sep. 2004. 98p.

2. Brasil. Ministério da Saúde. Manual para a Organização da Atenção Básica. Brasília: MS; 1999. p 6.

3. Campos GWS. Diretrizes para o ensino médico na rede básica de saúde. ABEM. [online]. Disponível em: http:/ / www.ufrgs.br/tramse/classicos/textos/2005/05/diretrizes-para-o-ensino-mde.htm

4. Brasil. Ministério da Educação. Conselho Nacional de Educação. Câmara de Educação Superior. Resolução CNE/CES n.4 de 7 de Novembro de 2001. Diretrizes Curriculares Nacionais do Curso de Graduação em Medicina. [online]. Diário Oficial da União, Brasília, 9 de novembro de 2001. Seção 1, p. 38. Disponível em http://portal.mec.gov.br/sesu/arquivos/pdf/0401Medicina.pdf>

5. Brasil. Ministério da Educação. Conselho Nacional de Educação. Câmara de Educação Superior. Resolução CNE/CES 3/2002. Diretrizes Curriculares Nacionais do Curso de Graduação em Odontologia. [online]. Diário Oficial da União, Brasília, 4 de março de 2002. Seção 1, p.10. Disponível em <http: / / portal.mec.gov.br / sesu / a r quivos / p d f /0302Odontologia.pdf>

6. Brasil. Ministério da Educação. Conselho Nacional de Educação. Câmara de Educação Superior. Resolução CNE/CES 3/2001. Diretrizes Curriculares Nacionais do Curso de Graduação em Enfermagem. [online]. Diário Oficial da União, Brasília, 9 de novembro de 2001. Seção1, p.37. Disponível em <http: / / portal.mec.gov.br / s e s u / a rquivos / pdf / 0301Enfermagem.pdf>
7. Brasil. Ministério da Saúde. Portaria 198/GM/MS. Diário Oficial da União nº 32/2004, Secção I.

8. Brasil. Ministério da Saúde. Pró-saúde: programa nacional de reorientação da formação profissional em saúde /Ministério da Saúde, Ministério da Educação. Brasília: Ministério da Saúde; 2005. 77 p. (Série C. Projetos, Programas e Relatórios).

9. Brasil 1988. Constituição da República Federativa do Brasil. Brasília: Senado; 1988. Cap. II, Seção II, Art. 200.

10. Ceccim RB. Educação Permanente em Saúde: desafio ambicioso e necessário. Interface comum saúde educ. 2005;9(16):161-162.

11. Blejmar B, Almeida M. Mudança organizacional e transformação social. In: A Educação dos profissionais de saúde na Amédica Latina. Teoria e prática de um movimento de mudança. Almeida M, Feuerwerker L, Llanos M. (Org.). São Paulo: Hucitec; 1999. p.17-45

\section{CONTRIBUIÇÃO DOS AUTORES}

Anete Trajman e Walria Toschi participaram da concepção do projeto e se responsabilizaram pela aprovação no comitê de ética, contato com diretores de unidades, análise dos dados e redação final do manuscrito. Diogo Tobias, Monique Venturi e Naima Assunção coletaram os dados em campo e participaram da análise dos resultados e da redação do manuscrito. Victoria Brant fez contribuições substanciais na redação da forma final do manuscrito e na análise crítica dos resultados.

Apoio: MV e DT são bolsistas do Programa Institucional de Iniciação Científica (Pibic) da UGF; NA e VB são bolsistas do $\mathrm{CNPq}$ (Pibic e produtividade); AT é colaboradora (key personnel) do projeto ICOHRTA FIC/NIH \# 5U2 R TW006883-03.

O presente estudo não teve financiamento para a sua realização.

\section{CONFLITO DE INTERESSES}

Declarou não haver.

\section{ENDEREÇO PARA CORRESPONDÊNCIA}

Anete Trajman

Rua Macedo Sobrinho, 74 - apto 203

Humaitá

CEP. 22271-080 - Rio de Janeiro - RJ

E-mail: atrajman@gmail.com 